( М.В. Шестакова ${ }^{1,2 *}$, А.С. Колбин ${ }^{3,4}$, О.И. Карпов ${ }^{5}$, Г.Р. Галстян ${ }^{1}$, А.Ю. Майоров ${ }^{1}$, М.А. Арепьева ${ }^{4}$, А.А. Курылев ${ }^{3}$, М.А. Проскурин ${ }^{4}$

'Национальный медицинский исследовательский центр эндокринологии, Москва

2Первый Московский государственный медицинский университет им. И.М. Сеченова, Москва

${ }^{3}$ Первый Санкт-Петербургский государственный медицинский университет имени академика И.П. Павлова

Минздрава России, Санкт-Петербург

${ }^{4}$ Санкт-Петербургский государственный университет, Санкт-Петербург

${ }^{5} \mathrm{AO}$ «Санофи Россия», Москва

ОБОСНОВАНИЕ. Своевременная диагностика сахарного диабета 2 типа (СД2) является актуальной задачей для начала лечения и предупреждения осложнений заболевания. Наиболее распространенным методом первичной диагностики является определение глюкозы крови натощак (ГНТ). Альтернативным методом выявления болезни является тест на гликированный гемоглобин $\left(\mathrm{HbA}_{1 c}\right)$, который дороже и потому применяется реже.

ЦЕЛЬ. Моделирование затрат на лечение СД2 во временной перспективе при использовании для диагностики теста на $\mathrm{HbA}_{1 \mathrm{c}}$ в сравнении с анализом ГНТ.

МЕтодЫ. Ретроспективный анализ агрегированных частей базы данных эпидемиологического российского исследования NATION у 810 больных с предиабетом и СД2, у которых есть результаты тестов на НbА 1 и ГНТ с определением чувствительности и специфичности каждого из них. Для клинико-экономической оценки использовали симуляционную модель исходов СД2 с дискретным ходом событий для горизонта 20 лет с определением прямых и непрямых расходов. Были рассмотрены три алгоритма лечения СД2 с точки зрения затрат для своевременно выявленного заболевания и запоздалого диагноза с использованием метформина, глифлозинов, глиптинов, агонистов рецепторов к глюкагоноподобному пептиду, базальных аналогов инсулина и их фиксированной комбинации.

РЕзУЛЬтАтЫ. Анализ ГНТ дает существенно больше отрицательных ответов в диагностике СД2, чем тест на НbА (77,4\% и 36,5\% соответственно). Однако при этом число ложноотрицательных результатов при ГНТ в 3 раза превышает таковое при определении $\mathrm{HbA}_{1 c}$. Тест на $\mathrm{HbA}_{1 c}$ в 3 раза более точно определяет нарушения углеводного обмена, чем анализ ГНТ. Своевременная диагностика с помощью данных о НbА 1 в сравнении с запоздалой по тесту ГНТ позволяет сократить общие расходы на лечение СД2 на 26,3-27,7\% в зависимости от рассмотренного алгоритма начатого лечения за счет прогнозируемого снижения числа макрососудистых осложнений вследствие эффективного контроля заболевания. Своевременная диагностика с использованием теста на $\mathrm{HbA}_{1 c}$ снижает вероятность инвалидизации на 21\% к 20-му году моделирования в сравнении с поздним выявлением заболевания с помощью ГНТ.

ЗАКЛЮчЕНИЕ. Тест на НbA нии (диспансеризации). Средние прямые и непрямые затраты на одного пациента в 20-летней перспективе, включающие стоимость как рассмотренных анализов, так и смоделированных осложнений Сд2, ниже при применении для первичной диагностики теста на $\mathrm{HbA}_{1 с}$ в сравнении с ГНТ.

КЛЮЧЕВЫЕ СЛОВА: сахарный диабет 2 mипа; тест на гликированный гемоглобин; тест на глюкозу крови натощак; анализ технологии здравоохранения

\title{
AN ECONOMIC VALUE OF THE GLYCATED HEMOGLOBIN TEST IN DIABETES MELLITUS TYPE 2 DIAGNOSIS
}

(c) Marina V. Shestakova1,2*, Alexey S. Kolbin³,4, Oleg I. Karpov5, Gagyk R. Galstyan', Aleksandr Y. Mayorov' , Maria A. Arepeva, Alexey A. Kurilev ${ }^{3}$, Maxim A. Proskurin ${ }^{4}$

'Endocrinology Research Centre, Moscow, Russia

2I.M. Sechenov First Moscow State Medical University, Moscow, Russia

${ }^{3}$ Pavlov First Saint Petersburg State Medical University, Saint-Petersburg, Russia

${ }^{4}$ Saint-Petersburg State University, Saint-Petersburg, Russia

${ }^{5}$ Sanofi Russia JSC, Moscow, Russia

BACKGROUND: Diagnostic of diabetes mellitus type 2 (DM2T) in time is very actual for treatment and prevention of potential complications of illness. Fasting blood glucose test (FBG) is a widely used method of primary DM2T diagnose. Glycated hemoglobin $\left(\mathrm{HbA}_{1 \mathrm{c}}\right)$ test is an alternative and used more rarely due to expensive. 
AIM: Modelling of comparative expenditures for DM2T control in cases of primary diagnostic by $\mathrm{HbA}_{1 \mathrm{c}}$ test or FBG test usage in 20-years horizon.

METHODS: Retrospective analysis of aggregated epidemiological Russian NATION study data in 810 patients with prediabetes and DM2T with both analysis performed, with sensitivity and specificity of each is detected. The simulation model of DM2T outcomes has been used for Health Technology assessment (direct and indirect costs of Diabetes control during 20 years). Three algorithms of the DM2T treatment were investigated for understanding of expenditures in case on diagnostic on-time and case of late verification with metformin, gliflozines, gliptins, Glucagon-like peptide-1 receptor agonists, basal insulin analogs and their combinations.

RESULTS: FBG test has more negative results for DM2T diagnostics in compare with $\mathrm{HbA}_{1 \mathrm{c}}$ analysis $(77,4 \%$ and $36,5 \%$ accordingly). Amount of false negative results in FBG test in 3 times more often occurred in comparison with $\mathrm{HbA}_{1 c^{\circ}}$ $\mathrm{HbA}_{1 \mathrm{c}}$ test in 3 times more precisely than FBG test for carbon metabolism abnormalities detection. Diagnostic in time with $\mathrm{HbA}_{1 \mathrm{c}}$ test in compare with late process of illness detection by FBG can cut common expenditures on $26,3-27,7 \%$ depending on treatment option due to macrovasular complications decreasing. Disability rate is expected decrease on $21 \%$ to 20 -th year in case of diagnostic with $\mathrm{HbA}_{1 \mathrm{c}}$ performing instead FBG test.

CONCLUSION: $\mathrm{HbA}_{1 \mathrm{C}}$ test has diagnostic advantages in compare with FBG test for primary investigation (dispanserization). Direct and indirect expenditures in average for one patient with DM2T on 20-years horizon including cost of drugs, analysis, complications, disabilities are less in case of diagnostic in time with $\mathrm{HbA}_{1 c}$ test in comparison with late diagnostics in case of FBG test execution.

KEYWORDS: diabetes mellitus type 2; $H b A_{1 C}$ test; fasting blood glucose test; health technology assessment

В настоящее время оценка уровня гликированного гемоглобина $\left(\mathrm{HbA}_{1 \mathrm{c}}\right)$ широко используется для контроля эффективности терапии сахарного диабета 2 типа (СД2). Тем не менее вопрос применения теста $\mathrm{HbA}_{1 с}$ для диагностики СД2 остается не до конца решенным, несмотря на то, что его оценка имеет ряд очевидных клинических преимуществ (не требует забора крови строго натощак и специальной подготовки к исследованию, обладает большой стабильностью получаемых результатов даже в периоды стрессов или интеркуррентных заболеваний, широко доступна в большинстве современных лабораторий) [1, 2]. Международный экспертный комитет заключил, что показатель $\mathrm{HbA}_{1 c} \geq 6,5 \%$ может быть использован в качестве порогового для скрининга на СД2. В отчете этого комитета также определены группы пациентов, для которых $\mathrm{HbA}_{1 c}$ может быть не показателен: пациенты с гемоглобинопатиями, дети и подростки, беременные [3]. Тем не менее в ряде исследований оценка $\mathrm{HbA}_{1 с}$ продемонстрировала свою эффективность в качестве метода диагностики СД2 с оптимальным уровнем чувствительности и специфичности [4, 5].

Немаловажным фактором, поддерживающим выбор теста на $\mathrm{HbA}_{1 c}$ в качестве метода диагностики СД2, является его связь с оценкой сердечно-сосудистых рисков. В сравнении с оценкой глюкозы плазмы натощак (ГНТ) $\mathrm{HbA}_{1 с}$ продемонстрировал эффективность не только в диагностике СД2, но и в определении риска сердечно-сосудистых заболеваний, общей смертности [6]. Также было показано, что именно оценка $\mathrm{HbA}_{1 c}$ приоритетна в клинической практике как метод выявления большего числа пациентов с высоким риском развития СД2 и сердечно-сосудистых заболеваний [7]. При этом подчеркивается значение глюкозотолерантного теста (ГТТ) как своего рода «третейского судьи» для верификации диагноза предиабета и СД2 [8]. Этот тест должен проводиться после повторного измерения $\mathrm{HbA}_{1 c^{\prime}}$ который повторит результаты отклонений от нормы, для того чтобы избежать ложноположительных реакций [9].

Несмотря на то что в еще в 2011 г. ВО3 одобрила возможность использования теста на $\mathrm{HbA}_{1 с}$ для диагностики СД2, а также очевидные преимущества данного метода, в рутинной практике обследование (в том числе диспансеризация и профилактические осмотры) взрослого населения все же проводится на основании определения уровня ГНТ. Одним из расхожих объяснений этой ситуации является большая стоимость теста на $\mathrm{HbA}_{1 c^{\prime}}$ которая в некоторых лабораториях превышает в 3-4 раза таковую для анализа глюкозы.

\section{ЦЕЛЬ}

Моделирование затрат на лечение СД2 во временной перспективе при использовании для диагностики теста на $\mathrm{HbA}_{1 с}$ в сравнении с анализом ГНТ.

\section{МЕТОДЫ}

Ретроспективный анализ агрегированных (без персонификации пациентов) частей базы данных эпидемиологического российского исследования NATION [10] по 810 респондентам (впервые выявленным больным СД2), у которых есть результаты трех анализов: $\mathrm{HbA}_{1 c^{\prime}}$ ГНТ и ГТТ как верификатора нарушений углеводного обмена. Диагностические критерии представлены в табл. 1.

Таблица 1. Референсные значения для определения нормы, предиабета и сахарного диабета 2 типа

\begin{tabular}{lccc}
\hline \multicolumn{1}{c}{ Тест } & Норма & Предиабет & Сахарный диабет 2 типа \\
\hline ГТТ, ммоль/л & $<7,8$ & $\geq 7,8$ и $<11,0$ & $\geq 11,1$ \\
Гликированный гемоглобин, \% & $<5,7$ & $\geq 5,7$ и $<6,5$ & $\geq 6,5$ \\
Глюкоза натощак (венозная кровь), ммоль/л & $<6,1$ & $\geq 6,1$ и $<7,0$ & $\geq 7,0$ \\
\hline
\end{tabular}

Примечания: ГТТ - глюкозотолерантный тест 
Определяли чувствительность, специфичность, PPV (positive predictive value, прогностическую ценность положительного результата, вероятность наличия заболевания при положительном (патологическом) результате теста; определяется как соотношение истинно положительных результатов, т.е. случаев, когда тест дает положительный прогноз при положительном результате по факту, к сумме истинно положительных и ложноположительных результатов, т.е. случаев, когда тест дает положительный прогноз при отрицательном результате по факту) [11] и NPV (negative predictive value, прогностическую ценность отрицательного результата, вероятность отсутствия заболевания при отрицательном (нормальном) результате теста; определяется как соотношение истинно отрицательных результатов, т.е. случаев, когда тест дает отрицательный прогноз при отрицательном результате по факту, к сумме истинно отрицательных и ложноотрицательных результатов, т.е. случаев, когда тест дает отрицательный прогноз при положительном результате по факту) тестов на $\mathrm{HbA}_{1 с}$ и ГНТ. Сравнивалось число ложноположительных и ложноотрицательных результатов по тестам диагностики $\mathrm{HbA}_{1 с}$ и ГНТ.

Для клинико-экономической оценки диагностики нарушений углеводного обмена с помощью рассматриваемых тестов использовали разработанную ранее симуляционную модель исходов СД2 с дискретным ходом событий в горизонте 20 лет [12]. Используемая в расчетах модель для каждого из 810 пациентов исходной выборки NATION на основании значений $\mathrm{HbA}_{1 c^{\prime}}$ уровней артериального давления (АД), массы тела, а также показателей липидограммы осуществляет расчет рисков развития во времени осложнений СД2. Данная модель позволяет с высокой долей вероятности прогнозировать развитие осложнений заболевания в зависимости от эффективности/неэффективности его контроля. При этом учитываются как эффект от проводимой сахароснижающей терапии, так и другие индикаторы, например, показатели липидограммы [13]. При клинико-экономической оценке вероятность развития осложнений СД2, полученных на основании прогноза модели, умножается на стоимость их терапии. Модель также учитывает непрямые затраты. Затраты на каждого пациента суммируются с последующим расчетом средних показателей.

Для каждой из групп пациентов, сформированных в зависимости от результатов определения $\mathrm{HbA}_{1 c^{\prime}}$ ГНТ и ГТТ, были сформулированы целевые значения $\mathrm{HbA}_{1 c}$ для контроля СД2, а также предложены три алгоритма терапии с горизонтом моделирования до 20 лет. Были рассмотрены три алгоритма лечения после диагностирования с точки зрения затрат.

1 алгоритм: 1 этап Метформин $\rightarrow 2$ эman Метформин + ингибиторы дипептидилпептидазы-4 (ИДПП4) $\rightarrow$ 3 эman Метформин + ИДПП4 + глифлозины $\rightarrow 4$ эman Метформин + ИДПП4 + глифлозины + аналог базального инсулина;

2 алгоритм: 1 эman Метформин $\rightarrow 2$ эman Метформин + глифлозины $\rightarrow 3$ эman Метформин + глифлозины + агонисты рецепторов к глюкагоноподобному пептиду (аГПП-1) $\rightarrow 4$ этап Метформин + глифлозины + аГПП-1 + аналог базального инсулина;
3 алгоритм: 1 эman Метформин $\rightarrow 2$ эman Метформин + аГПП-1 $\rightarrow 3$ этап - Метформин + фиксированная комбинация аналога базального инсулина (инсулин гларгин 100 ЕД/мл) + а ГПП-1 (ликсисенатид).

В модели были использованы показатели эффективности сахароснижающих средств и аналогов инсулина по контролю СД2, взятые из исследований [14-28].

Расчет стоимости средней суточной дозы, определенной по так называемой средней установленной суточной дозе (DDD, Defined Daily Dose) [29], представлен в табл. 2. При этом в случае наличия нескольких препаратов по одному международному непатентованному наименованию (МНH) рассчитывалась средняя предельная отпускная цена. Для препаратов, входящих в Перечень жизненно необходимых и важнейших лекарственных препаратов (ЖНВЛП), принимались во внимание зарегистрированные цены (основаны на государственном реестре лекарственных средств (ГРЛС)) [30]. Для препаратов, не входящих в ГРЛС, использовались оптовые цены Российского фармацевтического портала «ФармИндекс» [31], для комбинации инсулина гларгин 100 ЕД/мл и ликсисенатида использована стоимость, предоставленная производителем. Суточная стоимость рассчитана с учетом НДС и средней оптовой надбавки, которая, в свою очередь, высчитана на основании данных мониторинга за оптовыми ценами [32]. Для препаратов одной фармакологической группы высчитана средняя стоимость лечения в день.

Усредненная стоимость ГТТ - 700 руб., стоимость исследования ГНТ - 270 руб., определение $\mathrm{HbA}_{1 c}$ 650 руб. [33-35].

Стоимость терапии осложнений и непрямых затрат, связанных с их развитием, приведена в табл. 3. При расчете непрямых затрат исходили из значений недополученного валового внутреннего продукта (ВВП) в день, рассчитанного на основании средней заработной платы в РФ в 2018 г., равной 43724 руб./мес, или 1457,47 руб./день.

При расчетах непрямых затрат учитывали:

недополученный ВВП вследствие стойкой утраты трудоспособности из-за наступления инвалидности в трудоспособном возрасте;

- недополученный ВВП ввиду временной и стойкой утраты трудоспособности.

Расчет непрямых потерь ВВП проводили исходя из значения ВВП РФ на душу населения (1), а также среднего дневного заработка (2) (табл. 4).

Ниже представлены формулы расчета непрямых затрат:

$$
\text { ВВП (руб./чел./день) }=\frac{\text { ВВП РФ (руб.) }}{\text { Численность }} / 365 \text { дней (1) }
$$

ВВП (руб./чел./день)= (средняя зарплата 2017 г. + средняя зарплата 2018 г.) /2 /30 дней (2)

Для осуществления расчетов использовался Microsoft Excel.

\section{РЕЗУЛЬТАТЫ}

У 810 больных СД2 из базы данных NATION было проведено сопоставление диагностической значимости двух тестов - теста на $\mathrm{HbA}_{1 c}$ и ГНТ, «третейским судьей» в верификации диагноза выступал ГТТ. При одной 
Таблица 2. Суточная стоимость терапии сахароснижающими препаратами

\begin{tabular}{|c|c|c|c|c|}
\hline МHH & Упаковка & $\begin{array}{c}\text { Стоимость упаковки } \\
\text { без НДС (руб.) }\end{array}$ & DDD & $\begin{array}{c}\text { Средняя суточная } \\
\text { стоимость терапии } \\
\text { (руб.) }\end{array}$ \\
\hline Метформин* & 1000 мг №30 & 120,2 & $2000 \mathrm{Mг}$ & 9,36 \\
\hline Ингибиторы ДПП-4 & & - & - & 56,17 \\
\hline Ситаглиптин* & 100 мг №28 & 1305,03 & $100 \mathrm{Mr}$ & 56,39 \\
\hline Вилдаглиптин* & 50 мг №56 & 1294,90 & $100 \mathrm{Mr}$ & 55,95 \\
\hline Глифлозины & & - & - & 137,88 \\
\hline Дапаглифлозин* & 10 мг №30 & 2040,00 & $10 \mathrm{Mr}$ & 82,28 \\
\hline Эмпаглифлозин* & 10 мг №30 & 2378,70 & $17,5 \mathrm{мг}$ & 167,89 \\
\hline Канаглифлозин & 100 мг №30 & 2026,45 & $200 \mathrm{Mr}$ & 163,47 \\
\hline аГПП-1 & & - & - & 201,74 \\
\hline Ликсисенатид* & $\begin{array}{c}\text { 0,1 мг/мл } \\
\text { (20 мкг/доза), } 3 \text { мл №2 }\end{array}$ & 3933,00 & $0,02 \mathrm{Mr}$ & 158,63 \\
\hline Эксенатид & 0,25 мг/мл, 2,4 мл & 4830,58 & $0,015 \mathrm{Mг}$ & 146,12 \\
\hline Лираглутид & 6 мг/мл, 3 мл, №2 & 7450,00 & $1,2 \mathrm{Mr}$ & 300,48 \\
\hline $\begin{array}{l}\text { Инсулин гларгин + } \\
\text { ликсисенатид** }\end{array}$ & & & & 163,05 \\
\hline $\begin{array}{l}\text { Инсулин гларгин + } \\
\text { ликсисенатид }\end{array}$ & $\begin{array}{c}100 \text { ЕД/мл + } 33 \text { мкг/мл, } \\
3 \text { мл №3 }\end{array}$ & 2916,00 & $\begin{array}{c}40 \text { ЕД по инсулину } \\
\text { гларгин }\end{array}$ & 129,60 \\
\hline $\begin{array}{l}\text { Инсулин гларгин + } \\
\text { ликсисенатид }\end{array}$ & $\begin{array}{c}100 \text { ЕД/мл + } 50 \text { мкг/мл, } \\
\text { Змл №3 }\end{array}$ & 3654,00 & $\begin{array}{c}40 \text { ЕД по инсулину } \\
\text { гларгин }\end{array}$ & 196,50 \\
\hline $\begin{array}{l}\text { Базальные } \\
\text { аналоги инсулина }\end{array}$ & & & & 89,35 \\
\hline Инсулин гларгин* & $\begin{array}{c}100 \text { ЕД/мл, } 3 \text { мл, №5 } \\
300 \text { ЕД/мл, 1,5 мл, №3 }\end{array}$ & $\begin{array}{l}2686,16 \\
2588,33\end{array}$ & 40 ЕД & 73,48 \\
\hline Инсулин детемир* & 100 ЕД/мл, 3 мл, №5 & 2263,68 & 40 ЕД & 73,08 \\
\hline Инсулин деглудек* & 100 ЕД/мл, 3 мл, №5 & 3762,43 & 40 ЕД & 121,48 \\
\hline
\end{tabular}

Примечания: * препараты, включенные в Перечень ЖНВлП; ** включен в ЖНВЛП с 2020 г., представлена стоимость, которую производитель собирается зарегистрировать. МНН - международное непатентованное наименование; ДПП-4 - дипептидилпептидаза-4; аГПП-1 - агонисты глюкагоноподобного пептида 1; DDD - Defined Daily Dose (средняя установленная суточная доза); НДС - налог на добавленную стоимость.

Таблица 3. Стоимость гипогликемии и терапии осложнений сахарного диабета 2 типа* и непрямые затраты, связанные с их развитием

\begin{tabular}{|c|c|c|c|c|}
\hline Осложнение & Тариф & $\begin{array}{l}\text { Код тарифа } \\
\text { по КСГ }\end{array}$ & $\begin{array}{c}\text { Количество } \\
\text { дней временной } \\
\text { нетрудоспособности }\end{array}$ & $\begin{array}{l}\text { Непрямые затраты } \\
\text { в связи с ВУт }\end{array}$ \\
\hline Гипогликемия** & 59,55 & - & 0 & 0 \\
\hline Тяжелая гипогликемия** & 15180,00 & 231190 & 12 & 17489,64 \\
\hline Синдром диабетической стопы & 94626,00 & 321640 & 60 & 87448,2 \\
\hline $\begin{array}{l}\text { Острый инфаркт миокарда } \\
\text { (в т. ч. включая реабилитацию) }\end{array}$ & 313740,90 & $\begin{array}{l}\text { вСХ001-2 } \\
\text { и } 511551\end{array}$ & $23+25$ & 69958,56 \\
\hline ИБС, стенокардия напряжения & 37816,80 & 421170 & 14 & 20404,58 \\
\hline Сердечная недостаточность & 47844,70 & 291110 & 16 & 23319,52 \\
\hline $\begin{array}{l}\text { Острое нарушение мозгового } \\
\text { кровообращения (по ишемическому } \\
\text { типу) (в т. ч. включая реабилитацию) }\end{array}$ & 145987,80 & 261331 и 511600 & $18+30$ & 69958,56 \\
\hline $\begin{array}{l}\text { Примечания: ВУТ - временная утрата трудоспос } \\
\text { ральным тарифным соглашением (ГТС) с учетом } \\
\text { вызова скорой помощи или госпитализации пац } \\
\text { лагается, что пациент использует в среднем } 3 \text { тес } \\
\text { ного лечения гипогликемического синдрома, со } \\
2019 \text { год [36]). }\end{array}$ & лительности & $\begin{array}{l}\text { ечения по КСГ, указан } \\
\text { ется употреблением в } \\
\text { имостью } 19,85 \text { руб./шт) } \\
\text { 2нию №4 к КТС составЈ }\end{array}$ & $\begin{array}{l}\text { группы; * - тарифы рассчита } \\
\text { в ГТС [36]; }{ }^{* *} \text { - нетяжелая гv } \\
\text { цу продуктов с высоким сод } \\
\text { на каждый эпизод гипоглик } \\
\text { т } 15 \text { 180,00 руб. (Генерально }\end{array}$ & $\begin{array}{l}\text { ы в соответствии с Гене- } \\
\text { огликемия, не требует } \\
\text { ожанием глюкозы. Предпо- } \\
\text { мии. Стоимость стационар- } \\
\text { тарифное соглашение ОМС, }\end{array}$ \\
\hline
\end{tabular}


Таблица 4. Данные для расчетов непрямых затрат

\begin{tabular}{lc}
\hline \multicolumn{1}{c}{ Параметр } & Значение \\
\hline \multicolumn{2}{c}{ РВСчет по ВВП РФ } \\
Население РФ в 2018 г. & 1570 млрд руб. \\
ВВП на душу населения 2018 год, руб./чел./год & 146880432 \\
ВВП в день на душу населения 2018 год, руб./чел./день & 707113,68 руб. \\
\hline \multicolumn{2}{c}{ Расчет по средней дневной заработной плате } \\
\hline Средняя заработная плата в 2017 г. & 1937,30 руб. \\
Средняя заработная плата в 2018 г. & 37400,00 руб. \\
Средняя заработная плата в день & 42100,00 руб. \\
Возраст выхода на пенсию (мужчины) & 1325,00 руб. \\
Возраст выхода на пенсию (женщины) & 65 лет* \\
\hline
\end{tabular}

Примечания: * перспективные параметры. ВВП - внутренний валовый продукт; РФ - Российская Федерация.

и той же глюкозной нагрузке при выполнении ГТТ разница между минимальным и максимальным значениями уровня ГНТ составила 37\%, в то время как по тесту на $\mathrm{HbA}_{1 c}$ - только 19\% (рис. 1). При стандартизации данных выявлено, что при анализе ГНТ общее число отрицательных ответов было существенно больше, чем при анализе на $\mathrm{HbA}_{1 c}$ (77,4\% и 36,5\% соответственно). При этом число ложноотрицательных результатов при анализе ГНТ было в 3 раза выше, чем в случае анализа на $\mathrm{HbA}_{1 с}$ (табл. 5). Тест на $\mathrm{HbA}_{1 c}$ в 3 раза более точно определял нарушение углеводного обмена, чем тест ГНТ. Также отмечается, что частота и ложноположительных ответов по тесту на $\mathrm{HbA}_{1 c}$ была несколько выше. Отсюда можно сделать вывод о том, что тест ГНТ лучше определяет норму, но значительно хуже диагностирует нарушение углеводного обмена в сравнении с тестом на $\mathrm{HbA}_{1 c^{\prime}}$ что также подтверждают различные значения чувствительности этих тестов. Поэтому можно считать, что тест на $\mathrm{HbA}_{1 c}$ в большей степени отвечает требованию своевременной диагностики нарушений углеводного обмена, несмотря на его более высокую стоимость в сравнении с тестом ГНТ. Что касается большей частоты ложноположительных результатов, то, как мы покажем далее, затраты на них с лихвой перекрываются предупреждением различных макрососудистых осложнений у своевременно выявленных с помощью этого теста больных и ранним началом лечения СД2. Ложноотрицательный результат теста ГНТ в большом проценте случаев может приводить к тому, что заболевание не диагностируется в дебюте, пациента отпускают домой, когда он попадет в поле зрения врачей снова, неизвестно, возможно, через несколько лет. К этому времени у больного уже формируются осложнения, приводящие не только к ухудшению качества жизни, но и влекущие за собой расходы как здравоохранения и общества в целом, так и персональные. Было установлено, по данным исследования NATION, что половина выявленных больных не знали о наличии у них СД2, а результаты другого отечественного исследования показали, что только 25\% больных в группе пациентов с СД2 в возрасте 40-59 лет не имели осложнений, что как раз и является одним из последствий несвоевременного выявления заболевания [10].

Если бы мы основывались только на утилитарной сторонедела (себестоимость выполнения тестов), то пришли бы к выводу, что на 100 обследованных в случае анализа ГНТ было бы потрачено 27000 тыс. руб., при этом эффективность вложений, выраженная через коэффициент (отношение стоимости выявленных нарушений к ложным результатам), составила бы 18225 руб. / 8775 руб. = 2,08, а для теста на $\mathrm{HbA}_{1 с}$ такой же показатель был бы равен 33800 / $36200=0,93$. При таком подходе можно сделать вывод о том, что рациональнее было бы с эконо-

Таблица 5. Результаты сравнения анализов глюкозы крови натощак и гликированного гемоглобина для диагностики нарушений углеводного обмена $(\mathrm{n}=810)$

\begin{tabular}{lcc}
\hline \multicolumn{1}{c}{ Показатель } & \multicolumn{2}{c}{ Нарушение углеводного обмена (ПД + СД) } \\
\cline { 2 - 3 } & Глюкоза крови натощак & Гликированный гемоглобин \\
\hline Истинно положительные, $\mathrm{n}(\%)$ & $43(5,3)$ & $3131(16,2)$ \\
Ложноположительные, $\mathrm{n}(\%)$ & $140(17,3)$ & $261(32,2)$ \\
Истинно отрицательные, $\mathrm{n}(\%)$ & $504(62,2)$ & $35(4,3)$ \\
Ложноотрицательные, n (\%) & $123(15,2)$ & 79 \\
Чувствительность, \% & 26 & 41 \\
Специфичность, \% & 78 & 25 \\
PPV, \% & 23 & 88 \\
NPV, \% & 80 &
\end{tabular}

Примечания: ПД - предиабет; СД - сахарный диабет; PPV - positive predictive value (положительная предсказательная значимость, доля всех верных положительных прогнозов по отношению ко всем сделанным положительным прогнозам); NPV - negative predictive value (отрицательная предсказательная значимость, доля всех верных отрицательных прогнозов по отношению ко всем сделанным отрицательным прогнозам). 
A

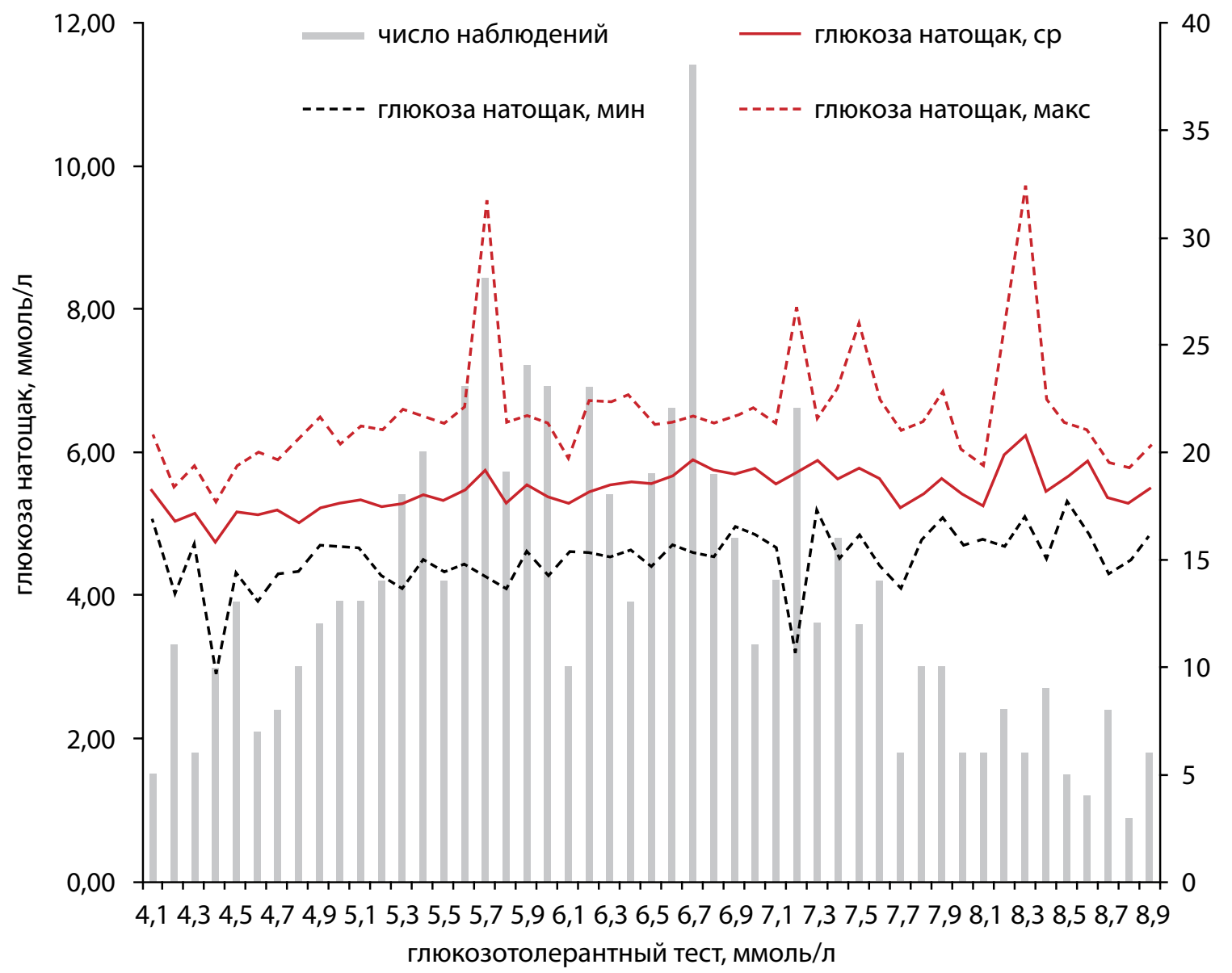

Б

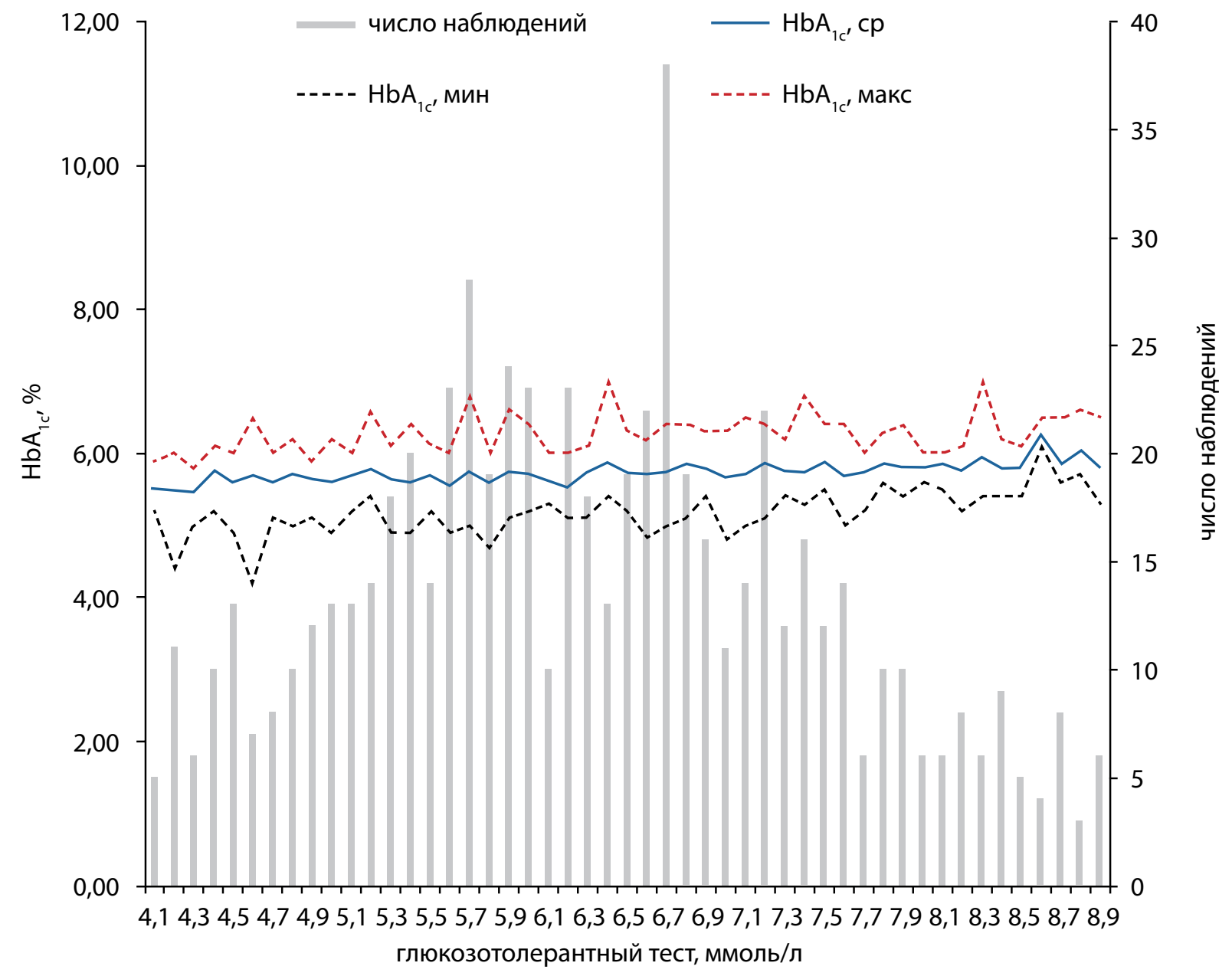

Рис. 1. Разброс значений глюкозы натощак (А) и гликированного гемоглобина (Б) при одинаковых уровнях глюкозной нагрузки. 
Таблица 6. Усредненные моделированные затраты на одного пациента на период 20 лет (руб.)

\section{Прямые медицинские затраты}

\begin{tabular}{|c|c|c|c|c|c|c|c|}
\hline \multirow[b]{2}{*}{ Анализ } & \multicolumn{3}{|c|}{ Прямые медицинские затраты } & \multicolumn{3}{|c|}{ Непрямые затраты } & \multirow[b]{2}{*}{ Итого } \\
\hline & $\begin{array}{c}\text { ТТГ и } \\
\text { анализ }\end{array}$ & $\begin{array}{c}\text { Затраты на } \\
\text { лекарственную } \\
\text { терапию }\end{array}$ & $\begin{array}{c}\text { Затраты на } \\
\text { купирование } \\
\text { осложнений }\end{array}$ & $\begin{array}{c}\text { Затраты ФСС } \\
\text { на выплату } \\
\text { пособий по } \\
\text { временной } \\
\text { нетрудоспособ- } \\
\text { ности }\end{array}$ & $\begin{array}{c}\text { Затраты } \\
\text { на } \\
\text { пособие } \\
\text { по } \\
\text { инвалид- } \\
\text { ности }\end{array}$ & $\begin{array}{c}\text { Недополученный } \\
\text { ВВП }\end{array}$ & \\
\hline \multicolumn{8}{|c|}{ Алгоритм лечения №1 } \\
\hline ГHТ & 970 & 1334967 & 2055039 & 1147391 & 842918 & 6361782 & 11743067 \\
\hline $\begin{array}{l}\text { Тест } \\
\mathrm{HbA}_{1 \mathrm{c}}\end{array}$ & 1350 & 1337961 & 1535999 & 699437 & 677697 & 4236760 & $\begin{array}{c}8489205 \\
(-27,7 \%)\end{array}$ \\
\hline \multicolumn{8}{|c|}{ Алгоритм лечения №2 } \\
\hline ГНТ & 970 & 2024048 & 2043527 & 1144341 & 836873 & 6340588 & 12390346 \\
\hline $\begin{array}{l}\text { Тест } \\
\mathrm{HbA}_{1 \mathrm{c}}\end{array}$ & 1350 & 2030298 & 1517362 & 697022 & 668476 & 4211201 & $\begin{array}{c}9125709 \\
(-26,3 \%)\end{array}$ \\
\hline \multicolumn{8}{|c|}{ Алгоритм лечения №3 } \\
\hline ГНТ & 970 & 1874122 & 2043942 & 1141274 & 837721 & 6326581 & 12224609 \\
\hline $\begin{array}{l}\text { Тест } \\
\mathrm{HbA}_{1 \mathrm{c}}\end{array}$ & 1350 & 1884696 & 1516309 & 687023 & 669439 & 4162164 & $\begin{array}{c}8920981 \\
(-27,0 \%)\end{array}$ \\
\hline
\end{tabular}

Примечания: ФСС - фонд социального страхования; ГНТ - глюкоза крови натощак; ТТГ - тест толерантности к глюкозе; ВВП - валовый внутренний продукт; $\mathrm{HbA}_{1 с}$ - гликированный гемоглобин.

мической точки зрения применять для диагностики тест на ГНТ вместо теста $\mathrm{HbA}_{11}$. Но такой вывод должен быть подтвержден клинико-экономическими результатами последствий диагностики, которая в одном случае в случае диагностики с помощью $\mathrm{HbA}_{1 с}$ - намного более, а в другом - ГНТ - менее точная.

При клинико-экономическом моделировании осуществляли прогнозирование вероятности осложнений СД2 во временном горизонте 20 лет, а также расчет затрат на их терапию условного пациента с характеристиками из базы данных NATION (табл. 6).

При моделировании осуществлялся расчет вероятности развития осложнений СД, ряд из которых являют- ся жизнеугрожающими. Были выбраны для включения в модель следующие осложнения, наиболее часто приводящие к инвалидизации пациента:

- ишемическая болезнь сердца, стенокардия напряжения;

- острый инфаркт миокарда;

- острое нарушение мозгового кровообращения;

- сердечная недостаточность;

- синдром диабетической стопы.

На рис. 2 представлена динамика вероятности развития инвалидности, которая представляет собой сумму вероятностей развития вышеуказанных осложнений СД (иными словами, на графике отражена вероятность

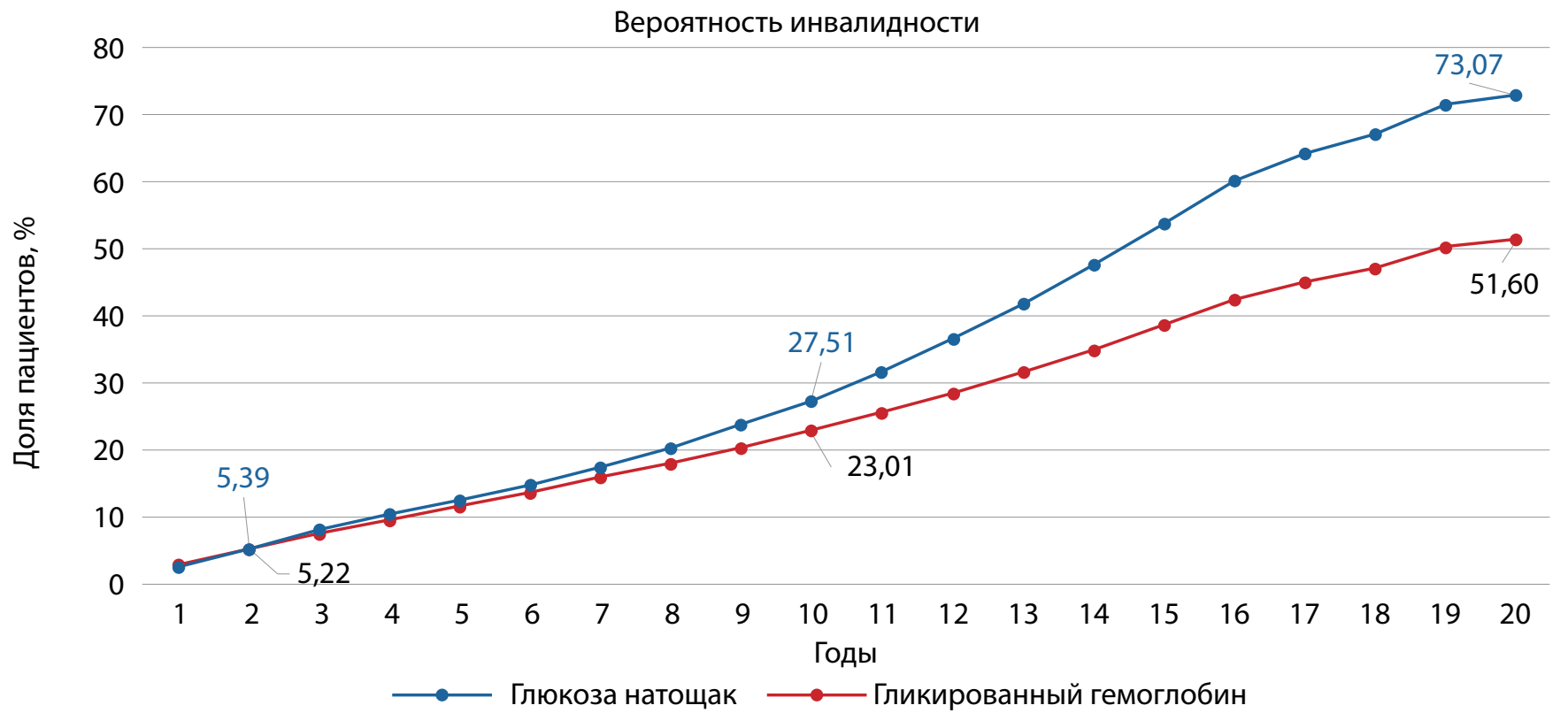

Рис. 2. Расчетная динамика количества пациентов с инвалидностью за 20-летний период (моделирование на основе базы NATІON). Глюкоза натощак - диагностика СД2 с помощью теста ГНТ; гликированный гемоглобин - диагностика с помощью определения НbА 
наступления любого или нескольких осложнений, приводящих к инвалидизации), изображенная нарастающим итогом отдельно для двух сравниваемых тестов. Как видно из данных, представленных на рис. 2, прирост вероятности наступления инвалидности при применении для диагностики СД2 теста ГНТ выше, чем при использовании $\mathrm{HbA}_{1 c^{\prime}}$ что объясняется меньшей чувствительностью этого теста для ранней диагностики СД2 и большим числом пациентов с недиагностированным диабетом.

Расчеты показывают, что при всех предложенных алгоритмах ведения больного со своевременно, с помощью теста на $\mathrm{HbA}_{1 c^{\prime}}$ выявленным заболеванием, расходы меньше, чем в случае задержки с диагностикой при применении теста ГНТ. Так, средние затраты на одного пациента при диагностике с помощью ГНТ-теста оказались по прогнозу выше по сравнению с диагностикой с определением $\mathrm{HbA}_{1 c}$ на 22,5-24,9\% в зависимости от рассмотренных алгоритмов. Наибольшая разница определяется за счет затрат на лечение осложнений (3 317908 руб./пациент для теста ГНТ и 2206003 руб./ пациент соответственно). Обращает внимание, что затраты на купирование осложнений при любом алгоритме в группе диагностики с помощью $\mathrm{HbA}_{1 с}$ ниже таковых в группе диагностики с помощью ГНТ.

Непрямые расходы в группе диагностирования СД2 с помощью теста на глюкозу составили 67,2-71,0\% числа всех затрат, в то время как в группе, диагностированной по $\mathrm{HbA}_{1 c^{\prime}}$ непрямые затраты занимали от $61,1 \%$ до $66,1 \%$. При этом наибольший процент от общих затрат отмечался по алгоритму 1, что может свидетельствовать о более эффективном вкладе лечения фиксированной комбинации «аГПП1 + базальный инсулин» в профилактику осложнений.

\section{ОБСУЖДЕНИЕ}

В настоящее время в большинстве стран мира, в том числе в России, при проведении скрининга для выявления нарушений углеводного обмена используются рекомендации ВО3, принятые В 1999 г. [37]. В Российской Федерации определение гликемии натощак (ГН) для диспансеризации, профилактических осмотров распространено в качестве скринингового теста. Однако результаты исследований по выявлению распространенности СД2 и других нарушений углеводного обмена в зависимости от критериев диагностики и метода постановки диагноза (гликемия натощак или оральный ГТТ (ОГТТ)) показали, что отказ от проведения ОГТТ ведет к снижению выявляемости ранних нарушений углеводного обмена. Это было продемонстрировано в ходе популяционных исследований, проведенных в Московской области [38]. Учитывая международный опыт в области диагностики СД, который исходит из позиции о высокой степени корреляции показателей $\mathrm{HbA}_{1 c}$ с показателями ГН и через 2 ч после нагрузки, а также тот факт, что $\mathrm{HbA}_{1 с}$ отражает состояние хронической гипергликемии и, соответственно, лучше прогнозирует риск развития сосудистых осложнений диабета, включение исследования уровня $\mathrm{HbA}_{1 с}$ для скрининга СД и других нарушений углеводного обмена могло бы значительно улучшить ситуацию со своевременным выявлением пациентов в Российской Федерации, особенно в группах повышенного риска по развитию заболева- ния (наследственный фактор, возраст, избыточная масса тела и др.).

Наши данные, полученные на отечественном эпидемиологическом материале (результаты широкомасштабного популяционного исследования NATION), совпадают с данными, полученными в других странах, где также показана большая диагностическая значимость теста на $\mathrm{HbA}_{1 с}$ в сравнении с тестом ГНТ. Так, в частности, при использовании теста на $\mathrm{HbA}_{1 с}$ распространенность предиабета и СД2 во Вьетнаме (проводилось с помощью квалифицированных экспертов на выборке 3523 человека) была 34,6\% и 9,7\% соответственно, а с помощью теста ГНТ - меньше - только 12,1\% и 6,3\% соответственно [39].

Экономическое моделирование динамики развития СД2 и его осложнений при своевременной (с помощью теста на $\mathrm{HbA}_{1 c}$ ) диагностике заболевания и сравнение его с диагностированием с помощью теста ГНТ с большим числом ложноотрицательных результатов, а следовательно, запоздалым началом лечения, проведено впервые. Теперь, кажется, ссылки на большую стоимость теста на $\mathrm{HbA}_{1 c^{\prime}}$ как показывает проведенное исследование, в условиях отечественного здравоохранения могут стать несостоятельными вследствие компенсации расходов на диагностику меньшими затратами на вовремя предупреждаемые осложнения заболевания в будущем. Особенно важно учитывать и снижение непрямых расходов вследствие эффективного контроля СД2. Тем не менее следует учитывать, что СД2 является неуклонно прогрессирующим заболеванием, поэтому даже высокоэффективные методы его контроля гипогликемизирующими средствами не могут предупредить его осложнения. Для более эффективной профилактики необходимы также липидснижающая терапия, нефропротективные, антигипертензивные и другие патогенетические средства.

\section{Ограничения исследования}

Анализ проведен на основании моделирования с использованием усредненных стоимостей лекарственных препаратов по фармакологическим группам и предложенных алгоритмов, число которых не ограничивается представленными в материале. При прогнозе осложнений и их стоимостей не учитывалось действие гиполипидемических, антигипертензивных, нефропротективных препаратов, назначение которых должно осуществляться в комплексе контроля СД2. Экономические расчеты сделаны с учетом тарифного соглашения.

\section{ЗАКЛЮЧЕНИЕ}

1. Определение $\mathrm{HbA}_{1 c}$ в сравнении с тестом ГНТ для первичной диагностики нарушений углеводного обмена (предиабет и СД2) характеризуется большей чувствительностью и меньшим числом ложноотрицательных результатов в отечественной популяции пациентов. Вследствие этого тест на $\mathrm{HbA}_{1 с}$ имеет диагностические преимущества перед анализом ГНТ при первичном обследовании (диспансеризации). Необходимо дальнейшее изучение этого вопроса, направленного на повышение диагностической значимости теста на $\mathrm{HbA}_{1 c^{\prime}}$ при сужении круга потенциальных больных, например, в группе (-ах) риска по нарушению углеводного обмена. 
2. Средние затраты на одного пациента, включающие как стоимость рассмотренных анализов, так и стоимость осложнений СД2, ниже при применении для первичной диагностики теста на $\mathrm{HbA}_{1 с}$ в сравнении с определением ГНТ при рассмотрении различных стратегий (алгоритмов) своевременной терапии до 27\% расходов в связи с предупреждением осложнений СД2 вовремя начатым лечением.

3. Моделирование с высокой долей вероятности показывает, что прирост наступления инвалидности при применении для диагностики теста ГНТ (с 27\% на 10-м году до 73\% к 20-му году) выше вследствие несвоевременной диагностики и наличия осложнений к моменту постановки диагноза, чем при использовании для этих же целей $\mathrm{HbA}_{1 с}$ (с 23\% на 10-м году до 52\% к 20-му году).

4. Прогнозируется, что непрямые расходы для всех рассмотренных в работе современных алгоритмов терапии были меньше у больных, у которых диагностирова- ние осуществлено с помощью теста на $\mathrm{HbA}_{1 c^{\prime}}$ при этом в общей структуре расходов эти затраты доминируют.

\section{ДОПОЛНИТЕЛЬНАЯ ИНФОРМАЦИЯ}

Источник финансирования. Исследование и подготовка публикации осуществлены на личные средства авторского коллектива.

Конфликт интересов. Профессор О.И. Карпов - сотрудник АО «Санофи Россия». Авторы декларируют отсутствие явных и потенциальных конфликтов интересов, связанных с публикацией настоящей статьи.

Участие авторов. Шестакова М.В. - разработка концепции, редактирование рукописи; Колбин А.С. - разработка концепции, план анализа, написание статьи; Карпов О.И. - разработка концепции, анализ данных, написание статьи; Галстян Г.Р. - редактирование статьи, Майоров А.Ю. - редактирование статьи; научное консультирование, Арепьева М.А. - математическое моделирование; Курылев А.А. - анализ полученных данных, написание статьи; Проскурин М.А. - обработка полученных данных, математическое моделирование.

\section{СПИСОК ЛИТЕРАТУРЫ | REFERENCES}

1. Дедов И.И., Шестакова М.В., Майоров А.Ю., и др. «Алгоритмы специализированной медицинской помощи больным сахарным диабетом» Под редакцией И.И. Дедова, М.В. Шестаковой, А.Ю. Майорова 9-й выпуск // Сахарный диабет. - 2019. - Т. 22. №1S1. - C. 1-144. [Dedov II, Shestakova MV, Mayorov AY, et al. Standards of specialized diabetes care. Edited by Dedov II, Shestakova MV, Mayorov AY. 9th edition. Diabetes mellitus. 2019;22(1S1):1-144. (In Russ.)] doi: https://doi.org/10.14341/DM221S1

2. American Diabetes Association. Classification and diagnosis of diabetes: standards of medical care in diabetes - 2019. Diabetes Care. 2019;42(Suppl 1):13-28. doi: https://doi.org/10.2337/dc19-S002

3. Nathan DM, Balkau B, Bonora E, et al. International expert committee report on the role of the A1c assay in the diagnosis of diabetes. Diabetes Care. 2009;32(7):1327-1334. doi: https://doi.org/10.2337/dc09-9033

4. Kumar PR, Bhansali A, Ravikiran M, et al. Utility of glycated hemoglobin in diagnosing type 2 diabetes mellitus: a communitybased study. J Clin Endocrinol Metab. 2010;95(6):2832-2835. doi: https://doi.org/10.1210/jc.2009-2433

5. Diabetes Prevention Program Research Group. $\mathrm{HbA} 1 \mathrm{c}$ as a predictor of diabetes and as an outcome in the Diabetes Prevention Program: a randomized clinical trial. Diabetes Care. 2015;38(1):51-58. doi: https://doi.org/10.2337/dc14-0886

6. Selvin E, Steffes MW, Zhu H, et al. Glycated hemoglobin, diabetes, and cardiovascular risk in nondiabetic adults. NEngl J Med. 2010;362(9):800-811. doi: https://doi.org/10.1056/NEJMoa0908359

7. Ackermann RT, Cheng YJ, Williamson DF, Gregg EW. Identifying adults at high risk for diabetes and cardiovascular disease using hemoglobin A1c National Health and Nutrition Examination Survey 2005-2006. Am J Prev Med. 2011;40(1):11-17 doi: https://doi.org/10.1016/j.amepre.2010.09.022

8. Gonzalez A, Deng Y, Lane A, et al. Impact of mismatches in $\mathrm{HbA}_{1 c}$ vs glucose values on the diagnostic classification of diabetes and prediabetes. Diabet Med. 2019. doi: https://doi.org/10.1111/dme.14181

9. Bachmann $\mathrm{MO}$, Lewis $\mathrm{G}$, John WG, et al. Determinants of diagnostic discordance for non-diabetic hyperglycaemia and Type 2 diabetes using paired glycated haemoglobin measurements in a large English primary care population: cross-sectional study. Diabet Med. 2019;36(11):1478-1486. doi: https://doi.org/10.1111/dme.14111

10. Дедов И.И., Шестакова М.В., Галстян Г.Р. Распространенность сахарного диабета 2 типа у взрослого населения России (исследование NATION) // Сахарный диабет. - 2016. - T. 19. №2. - C. 104-112. [Dedov II, Shestakova MV, Galstyan GR. The prevalence of type 2 diabetes mellitus in the adult population of Russia (NATION study). Diabetes mellitus. 2016;19(2):104-112. (In Russ.)] doi: https://doi.org/10.14341/DM2004116-17

11. Altman DG, Bland JM. Diagnostic tests 2: predictive values. BMJ. 1994;309(6947):102. doi: https://doi.org/10.1136/bmj.309.6947.102
12. Колбин А.С., Хмельницкий О.К., Курылев А.А., и др. Первый в России опыт построения симуляционной модели исходов сахарного диабета 2 типа с дискретным моделированием событий. Клиникоэкономическая экспертиза // Фармакоэкономика. - 2013. T. 6. - №2. - C. 24-31. [Kolbin AS, Khmelnitskiy OK, Kurylev AA, et al. First Russian type 2 diabetes mellitus simulation model with discreet events modeling. Health-economic analysis. Pharmacoeconomics. 2013;6(2):24-31. (In Russ.)]

13. Х Хмельницкий О.К., Колбин А.С., Курылев А.А. Моделирование медицинских и экономических исходов сахарного диабета // Заместитель главного врача. - 2013. - №8. - С. 94-105. [Khmel'nitskiy OK, Kolbin AS, Kurylev AA. Modelirovaniye meditsinskikh i ekonomicheskikh iskhodov sakharnogo diabeta. Zamestitel' glavnogo vracha. 2013;(8):94-105. (In Russ.)]

14. Domecq JP. Clinical review: drugs commonly associated with weight change: a systematic review and meta-analysis. J Clin Endocrinol Metab. 2015;100(2):363-370. doi: https://doi.org/10.1210/jc.2014-3421

15. Ma J, Liu LY, Wu PH, et al. Comparison of metformin and repaglinide monotherapy in the treatment of new onset type 2 diabetes mellitus in China. J Diabetes Res. 2014;2014:1-6. doi: https://doi.org/10.1155/2014/294017

16. Richter B, Bandeira-Echtler E, Bergerhoff K, Lerch CL. Dipeptidyl peptidase-4 (DPP-4) inhibitors for type 2 diabetes mellitus. Cochrane Database Syst Rev. 2008;(2):CD006739. doi: https://doi.org/10.1002/14651858.CD006739.pub2

17. Foley JE, Jordan J. Weight neutrality with the DPP-4 inhibitor, vildagliptin: mechanistic basis and clinical experience. Vasc Health Risk Manag. 2010;6:541-548. doi: https://doi.org/10.2147/vhrm.s10952

18. Lambers Heerspink HJ, de Zeeuw D, Wie L, et al. Dapagliflozin a glucose-regulating drug with diuretic properties in subjects with type 2 diabetes. Diabetes Obes Metab. 2013;15(9):853-862. doi: https://doi.org/10.1111/dom.12127

19. Roden M, Weng J, Eilbracht J, et al. Empagliflozin monotherapy with sitagliptin as an active comparator in patients with type 2 diabetes: a randomised, double-blind, placebo-controlled, phase 3 trial. Lancet Diab Endocrinol. 2013;1(3):208-219. doi: https://doi.org/10.1016/S2213-8587(13)70084-6

20. Bode B, Stenlöf $K$, Harris S, et al. Long-term efficacy and safety of canagliflozin over 104 weeks in patients aged $55-80$ years with type 2 diabetes. Diabetes Obes Metab. 2015;17(3):294-303. doi: https://doi.org/10.1111/dom.12428

21. Robinson LE, Holt TA, Rees $K$, et al. Effects of exenatide and liraglutide on heart rate, blood pressure and body weight: systematic review and meta-analysis. BMJ Open. 2013;3(1):e001986. doi: https://doi.org/10.1136/bmjopen-2012-001986

22. Rosenstock J, Davies M, Home PD, et al. A randomised, 52-week, treat-to-target trial comparing insulin detemir with insulin glargine when administered as add-on to glucose-lowering drugs in insulinnaive people with type 2 diabetes. Diabetologia. 2008;51(3):408-416. doi: https://doi.org/10.1007/s00125-007-0911-x 
23. Колбин А.С. Фармакоэкономика базально-болюсной инсулинотерапии сахарного диабета 2 типа // Клиническая фармакология и терапия. - 2011. - T. 20. — №1. - С. 92-96. [Kolbin AS. Pharmacoeconomic of basal-bolus insulin therapy in patients with type 2 diabetes. Klinicheskaya farmakologiya i terapiya. 2011;20(1):92-96. (In Russ.)]

24. Колбин А.С., Мосикян А.А., Курылев А.А., и др. Клиникоэкономический анализ ликсисенатида при сахарном диабете 2 типа // Качественная клиническая практика. — 2015. — №4. C. 53-63. [Kolbin AS, Mosikyan AA, Kurylev AA, et al. Economic analysis of lixisenatide in diabetes mellitus type 2. Kachestvennaya klinicheskaya praktika. 2015;(4):53-63. (In Russ.)]

25. Rosenstock J, Aronson R, Grunberger G, et al. Benefits of lixilan, a titratable fixed-ratio combination of insulin glargine plus lixisenatide, versus insulin glargine and lixisenatide monocomponents in type 2 diabetes inadequately controlled on oral agents: The lixilan-O randomized trial. Diabetes Care. 2016;39(11):2026-2035. doi: https://doi.org/10.2337/dc16-0917

26. Rosenstock J, Cheng A, Ritzel R, et al. More similarities than differences testing insulin glargine 300 Units $/ \mathrm{mL}$ versus insulin degludec 100 Units/mL in insulin-naive type 2 diabetes: the randomized head-to-head BRIGHT trial. Diabetes Care. 2018;41(10):2147-2154. doi: https://doi.org/10.2337/dc18-0559

27. Недогода С.В., Саласюк А.С., Барыкина И.Н., и др. Фармакоэкономическая оценка фиксированной комбинации инсулина гларгин и ликсисенатида при сахарном диабете 2 типа // Качественная клиническая практика. - 2019. — №3. - C. 13-22. [Nedogoda SV, Salasyuk AS, Barikina IN, et al. Economic evaluation of the fixed combination of insulin glargine and lixisenatide in diabetes mellitus type 2 . Kachestvennaya klinicheskaya praktika. 2019;(3):13-22. (In Russ.)] doi: https://doi.org/10.24411/2588-0519-2019-10079

28. Зырянов С.К., Дьяков И.Н. Экономические перспективы эффективного контроля сахарного диабета 2-го типа фиксированной комбинацией аналога базального инсулина и агониста рецепторов к глюкагоноподобному пептиду // Качественная клиническая практика. - 2019. - №2. - C. 4-14. [Zyryanov SK, Dyakov IN. Economic perspectives for effective control of diabetes mellitus type 2 with fixed combination of basal insulin analog and GLP-1 agonist. Kachestvennaya klinicheskaya praktika. 2019;(2):4-14. (In Russ.)] doi: https://doi.org/10.24411/2588-0519-2019-10068

29. WHO Collaborating Centre for Drug Statistics Methodology. ATC/DDD Index 2019. Available from: http://www.whocc.no/atc_ddd_index/

30. Государственный реестр лекарственных средств [интернет]. Безопасность лекарственных препаратов. [Gosudarstvennyy reyestr lekarstvennykh sredstv [Internet]. Bezopasnost' lekarstvennykh preparatov. (In Russ.)]. Доступно по: http://grls.rosminzdrav.ru. Ссылка активна на 10.10.2019.
31. ФармИндекс [интернет]. Оптовые цены для препаратов, не входящих в ГРЛС. [Farmlndeks [Internet]. Optovyye tseny dlya preparatov, ne vkhodyashchikh v GRLS. (In Russ.)]. Доступно по: http://pharmindex.ru. Ссылка активна на 10.10.2019.

32. Приказ ФАС России от 31.07.2019 № 1049/19 «О внесении изменений в сводный план проведения плановых проверок федеральных органов государственной власти, органов государственной власти субъектов Российской Федерации, органов местного самоуправления Федеральной антимонопольной службы на 2019 год». [Order № 1049/19 Federal Antimonopoly service of Russia «O vnesenii izmeneniy $v$ svodnyy plan provedeniya planovykh proverok federal'nykh organov gosudarstvennoy vlasti, organov gosudarstvennoy vlasti sub"yektov Rossiyskoy Federatsii, organov mestnogo samoupravleniya Federal'noy antimonopol'noy sluzhby na 2019 god»; dated 2019 July 31. (In Russ.)]. Доступно по: https://fas.gov.ru/documents/685845. Ссылка активна на 10.10.2019.

33. Лабораторная служба Helix [интернет]. Прайс-лист. [Laboratornaya sluzhba Helix [Internet]. Price list. (In Russ.)]. Доступно по: http://www.helix.ru. Ссылка активна на 10.10.2019.

34. ФГБУ «НМИЦ эндокринологии» Минздрава России [интернет]. Прайс-лист. [FGBU «NMITS endokrinologii» Minzdrava Rossii [Internet]. Price list. (In Russ.)]. Доступно по: https://www.endocrincentr.ru/. Ссылка активна на 10.10.2019.

35. Лабораторная служба Invitro [интернет]. Прайс-лист. [Laboratornaya sluzhba Invitro [Internet]. Price list. (In Russ.)]. Доступно по: http://www.invitro.ru. Ссылка активна на 10.10.2019.

36. Официальный сайт Территориального фонда ОМС СанктПетербурга [интернет]. Генеральное тарифное соглашение OMC, 2019 год. [Ofitsial'nyy sayt Territorial'nogo fonda OMS Sankt-Peterburga [Internet]. General'noye tarifnoye soglasheniye OMS, 2019 god. (In Russ.)]. Доступно по: http://www.spboms.ru. Ссылка активна на 10.10.2019.

37. World Health Organization. Definition, diagnosis and classification of diabetes mellitus and its complications: report of a WHO consultation. Part 1. Diagnosis and classification of diabetes mellitus. WHO, Geneva; 1999. Available from: https://apps.who.int/iris/handle/10665/66040

38. Древаль А.В., Мисникова И.В., Барсуков И.А., и др. Распространенность сахарного диабета 2 типа и других нарушений углеводного обмена в зависимости от критериев диагностики // Сахарный диабет. - 2010. - Т. 13. - №1. C. 116-121. [Dreval'AV, Misnikova IV, Barsukov IA, et al. Prevalence of type 2 diabetes mellitus and other abnormalities of carbohydrate metabolism depending on diagnostic criteria. Diabetes mellitus. 2010;13(1):116-121. (In Russ.)]

39. Ho-Pham LT, Nguyen UD, Tran TX, Nguyen TV. Discordance in the diagnosis of diabetes: Comparison between $\mathrm{HbA1C}$ and fasting plasma glucose. PLoS One. 2017;12(8):e0182192. doi: https://doi.org/10.1371/journal.pone.0182192

\section{ИНФОРМАЦИЯ ОБ АВТОРАХ [AUTHORS INFO]}

*Шестакова Марина Владимировна, д.м.н., профессор, академик PAH [Marina V. Shestakova, MD, PhD, Professor]; адрес: Россия, 117036 Москва, ул. Дм. Ульянова, д. 11 [address: 11 Dm. Ulyanova street, 117036 Moscow, Russian Federation]; ORCID: https://orcid.org/0000-0002-5057-127X; eLibrary SPIN: 7584-7015; e-mail: nephro@endocrincentr.ru

Колбин Алексей Сергеевич, д.М.Н., профессор [Alexey S. Kolbin, MD, PhD, Professor]; ORCID: https://orcid.org/0000-0002-1919-2909; eLibrary SPIN: 7966-0845: e-mail: alex.kolbin@mail.ru

Карпов Олег Ильич, д.М.Н., профессор [Oleg I. Karpov, MD, PhD, Professor]; ORCID: https://orcid.org/0000-0002-9370-5020; e-mail: oikar2012@gmail.com

Галстян Гагик Радикович, д.М.H., профессор [Gagik R. Galstyan, MD, PhD, Professor];

ORCID: https://orcid.org/0000-0001-6581-4521; eLibrary SPIN: 9815-7509; e-mail: galstyangagik964@gmail.com

Майоров Александр Юрьевич, д.м.н., профессор [Alexandr Y. Mayorov, MD, PhD, Professor];

ORCID: https://orcid.org/0000-0001-5825-3287; eLibrary SPIN: 4275-7779; e-mail: education@endocrincentr.ru

Арепьева Мария Александровна [Maria A. Arepeva]; ORCID: https://orcid.org/0000-0001-7923-1167;

e-mail: arepeva.maria@gmail.com

Курылев Алексей Александрович, к.M.н. [Alexey A. Kurilev, MD, PhD];

ORCID: https://orcid.org/0000-0003-3031-4572; eLibrary SPIN: 4470-7845; e-mail: alexey-kurilev@yandex.ru

Проскурин Максим Александрович [Maxim A. Proskurin];

ORCID https://orcid.org/0000-0002-9468-0953; eLibrary SPIN: 7406-2352; e-mail: proskurin.m@gmail.com 


\section{ЦИТИРОВАТЬ:}

Шестакова М.В., Колбин А.С., Карпов О.И., Галстян Г.Р., Майоров А.Ю., Арепьева М.А., Курылев А.А., Проскурин М.А. Экономическая ценность определения гликированного гемоглобина для диагностики сахарного диабета 2 типа // Сахарный диабет. — 2019. — T. 22. — №6. — С. 504-514. doi: https://doi.org/10.14341/DM12205

\section{TO CITE THIS ARTICLE:}

Shestakova MV, Kolbin AS, Karpov OI, Galstyan GR, Mayorov AY, Arepeva MA, Kurylev AA, Proskurin MA. An economic value of the glycated hemoglobin test in diabetes mellitus type 2 diagnosis. Diabetes Mellitus. 2019;22(6):504-514. doi: https://doi.org/10.14341/DM12205 